Glennie Richard ORCID iD: 0000-0003-3806-4280

\title{
Open population maximum likelihood spatial capture-recapture
}

\author{
R. Glennie ${ }^{1, *}$, D. L. Borchers ${ }^{1}$, M. Murchie ${ }^{1}$, B. J. Harmsen ${ }^{2,3}$, and R. J. Foster ${ }^{2,3}$
}

${ }^{1}$ University of St Andrews, Center for Research into Ecological and Environmental modeling, St Andrews, Fife, UK

1

${ }^{2}$ University of Belize, Environmental Research Institute, Belmopan, Belize

${ }^{3}$ Panthera, 8 West 40th Street, 18th Floor, New York, United States of America

*email:rg374@st-andrews.ac.uk

\begin{abstract}
SummarY: Open population capture-recapture models are widely used to estimate population demographics and abundance over time. Bayesian methods exist to incorporate open population modeling with spatial capturerecapture, allowing for estimation of the effective area sampled and population density. Here, open population spatial capture-recapture is formulated as a hidden Markov model, allowing inference by maximum likelihood for both Cormack-Jolly-Seber and Jolly-Seber models, with and without activity center movement. The method is applied to a twelve-year survey of male jaguars (Panthera onca) in the Cockscomb Basin Wildlife Sanctuary, Belize, to estimate survival probability and population abundance over time. For this application, inference is shown to be biased when assuming activity centers are fixed over time, while including a model for activity center movement provides negligible bias and nominal confidence interval coverage, as demonstrated by a simulation study. The hidden Markov model approach is compared with Bayesian data augmentation and closed population models for this application. The method is substantially more computationally efficient than the Bayesian approach and provides a lower root-mean-square error in predicting population density compared to closed population models.
\end{abstract}

KEY WORDS: hidden Markov model; open population; Panthera onca; population density; spatial capturerecapture; survival.

This article has been accepted for publication and undergone full peer review but has not been through the copyediting, typesetting, pagination and proofreading process, which may lead to differences between this version and the Version of Record. Please cite this article as doi:10.1111/biom.13078

This article is protected by copyright. All rights reserved. 


\section{INTRODUCTION}

\section{Introduction}

Many capture-recapture surveys (Otis et al., 1978) are conducted over time periods where the surveyed population undergoes change: individuals are born, immigrate, emigrate, or die. For these methods, each individual must have a unique mark that allows detectors to record encounters with them, creating their capture history. Two widely used statistical models for open populations, the Cormack-Jolly-Seber (CJS) (Cormack, 1964; Seber, 1965) and Jolly-Seber (JS) (Jolly, 1965; Seber, 1965) models, use these capture histories. For CJS models, inference is restricted to those individuals whose capture histories were recorded: marked individuals. From this, the apparent survival and detectability of marked individuals can be estimated. Only apparent survival is estimable since animals that die and those that permanently emigrate are indistiguishable from their capture histories. JS models, by assuming marked and unmarked individuals are exchangeable, extend inference to the entire population: estimating population size over time, recruitment (birth and immigration) rate, and survival rate.

Neither CJS nor JS incorporate the spatial component inherent in capture-recapture: individuals that range closer to a detector are more likely to be captured by that detector. Spatial capture-recapture (SCR) methods (Efford, 2004; Borchers and Efford, 2008) do use detector locations to estimate detection probability over space. This provides a rigorous estimate of the effective area sampled and population density.

SCR provides an efficient and flexible framework for closed population capture-recapture inference. Each individual is associated with a location in space, its activity center. The farther an activity center is from a detector, the less likely it is that the individual is captured by that detector. This activity center is a latent variable: it is unobserved. Maximum likelihood SCR modeling (Borchers and Efford, 2008) is achieved by numerical integration, averaging over all possible activity centers in the survey region. Bayesian SCR models (Royle et al., 2013) obtain inference by sampling activity centers within a Markov chain Monte Carlo (MCMC) algorithm, using the full joint likelihood of detection parameters and activity centers. Alternatively, Bayesian inference can be obtained from the semi-complete data likelihood where integration over activity centers is achieved by quadrature (King et al., 2016).

Existing open population SCR models (Gardner et al., 2010; Royle et al., 2013), which are extensions of CJS and JS, rely on a Bayesian approach and the full joint likelihood of detection parameters, activity centers, and life histories. Each individual has a latent life history that is sampled within an MCMC algorithm. For JS, data augmentation is used: a super-population, of pre-determined size, is sampled where some individuals are This article is protected by copyright. All rights reserved. 
born, and so contribute to the estimated population size, and some are never born. The super-population is chosen to ensure it exceeds the size of the true population and the MCMC sampling is used to infer the distribution of the population size. This method is computationally demanding and, in the formulation of Gardner et al. (2010), ties the interpretation of recruitment parameters to the size of the super-population; this dependence on the super-population size has been removed by Chandler and Clark (2014), though at the cost of further computation. No methods exist to fit open population SCR models by maximum likelihood; in particular, no algorithm other than MCMC has been used to average over all possible life histories.

In this paper, open population SCR is formulated as a hidden Markov model (HMM) (Zucchini et al., 2016), allowing for inference to be drawn by maximum likelihood and marginalization over all life histories to be done exactly. A HMM can be described by two processes: a hidden process and an observation process. The hidden process is a Markov chain of discrete states; the observations comprise a time series that depends on this hidden process: at each time, an observation depends only on the current state of the hidden process, and conditional on these hidden states, observations are independent. For open population SCR, the hidden process is the life history of the individual and the observations are the capture records (Figure 1). HMM methodology provides an efficient algorithm to average over all possible life histories. Combined with the extant numerical integration over activity centers, this allows both SCR CJS and JS models to be fit by maximum likelihood. Also, it allows for a semi-complete data likelihood to be computed and Bayesian inference obtained more efficiently.

\subsection{Motivation}

The JS method is applied to a camera trap survey of male jaguars (Panthera onca) in the Cockscomb Basin Wildlife Sanctuary, Belize (Harmsen et al., 2017). The survey was repeated for twelve years, between 2002 and 2015, during dry season months, between January and July. A key question for this population is how population size changes over time and what demographic processes drive this change. The method presented provides SCR estimates of population density, identifies which demographic rates are responsible for population change, and provides a means of testing whether these rates vary over time.

Finally, a simulation study is conducted to investigate the effect of small sample size, due to low population density or low detectability, on maximum likelihood estimates. The This article is protected by copyright. All rights reserved. 
simulation is based on the jaguar survey and estimates the bias and confidence interval coverage for each estimated parameter.

\section{Methods}

Consider a capture-recapture survey with $K$ occasions and $J$ detectors. During the survey, $n$ individuals are detected and uniquely marked, or identified to have some natural unique mark. Unique marks are used to recognise whether an individual was detected on each occasion or not: the capture history. Individual $i$ has a capture history $\boldsymbol{\Omega}_{i}$, a $J \times K$ matrix, with $k^{\text {th }}$ column $\boldsymbol{\omega}_{i,, k}$ and $(j, k)^{\text {th }}$ entry $\omega_{i, j, k}$. The entries of the capture history depend on the type of detector used: $\omega_{i, j, k}=0,1$ is binary for detectors that physically trap individuals or only register whether the individual was captured, or not, on each occasion (e.g. DNA hair traps); alternatively, $\omega_{i, j, k}$ can be the number of times an individual is encountered by the detector, if this is recorded (e.g. with cameras). The collection of all capture histories is denoted $\Omega$.

During each occasion, each individual is associated with a latent activity center in twodimensional space. Let $\boldsymbol{x}_{i, k}$ denote the activity center of individual $i$ during occasion

$k$ and let $\boldsymbol{x}_{i, 1}$ be termed the initial activity center of individual $i$. The activity centers are the spatial component of the model: each is the point in two-dimensional space that represents the average location of the individual over an occasion. In each occasion, the probability that an individual is detected on a particular detector is a decreasing function of the distance between this detector and the individual's activity center: those individuals that spend, on average, more time far away from the detector are detected less often than those that spend their time at a closer distance.

In open population capture-recapture, each individual has a life history, which is unobserved. On each occasion $k$, individual $i$ has one of three possible life states, $s_{i, k}$ : unborn, alive, or dead. Individuals who will be born or newly immigrate into the study area on an occasion after occasion $k$ are said to be in the 'unborn' state; individuals that are alive and present in the survey region on occasion $k$ are in the 'alive' state; finally, those individuals that died or permanently emigrated from the study area before occasion $k$ are said to be in the 'dead' state.

Here, the aim is to incorporate both SCR and open population capture-recapture into a single, tractable likelihood.

This article is protected by copyright. All rights reserved. 


\subsection{Detection model}

In a particular occasion, the probability that an individual is detected, and the number of times the individual is detected by each detector, depends on the individual's activity center and life state. This can be described by an encounter rate model where $\lambda_{j, k}(\boldsymbol{x}, s)$ is the mean number of captures of an individual with activity center $\boldsymbol{x}$ and life state $s$ during occasion $k$ at detector $j$. Clearly, individuals that are yet to be born and those who have already died cannot be detected: $\lambda_{j, k}(\boldsymbol{x}, s)=0$ for $s=$ unborn, dead. For those individuals that are alive on occasion $k, \lambda_{j, k}(\boldsymbol{x}$, alive), can be specified, for example, as a half-normal (or many other possible functional forms can be used, see Efford (2012)). Alternatively, one may specify the probability that an individual with activity center $\boldsymbol{x}$ and life state $s$ is seen at all in occasion $k$ by detector $j, p_{j, k}(\boldsymbol{x}, s)$, for example as half-normal, and then derive the encounter rate through the relationship $p_{j, k}(\boldsymbol{x}, s)=1-\exp \left\{-\lambda_{j, k}(\boldsymbol{x}, s)\right\}$.

Given the mean encounter rate, the probability of the observed capture record on each occasion can be stated. If capture records are binary, only a record of whether the animal was seen or not seen in each occasion is made, then the probability is $\left[\omega_{i, j, k} \mid \boldsymbol{x}_{i, k}, s_{i, k}\right]=p_{i, j, k}^{\omega_{i, j, k}}\left(1-p_{i, j, k}\right)^{1-\omega_{i, j, k}}$, where $p_{i, j, k}=p_{j, k}\left(\boldsymbol{x}_{i, k}, s_{i, k}\right)$, for brevity. If capture records are counts, which are all assumed to be independently Poisson distributed, then $\left[\omega_{i, j, k} \mid \boldsymbol{x}_{i, k}, s_{i, k}\right]=\lambda_{i, j, k}^{\omega_{i, j, k}} \exp \left(-\lambda_{i, j, k}\right) / \omega_{i, j, k}$ !, where $\lambda_{i, j, k}=\lambda_{j, k}\left(\boldsymbol{x}_{i, k}, s_{i, k}\right)$, for brevity.

The probability of the entire capture record on occasion $k$, assuming detectors and detections are independent, is thus $\left[\boldsymbol{\omega}_{i, \cdot, k} \mid \boldsymbol{x}_{i, k}, s_{i, k}\right]=\prod_{j=1}^{J}\left[\omega_{i, j, k} \mid \boldsymbol{x}_{i, k}, s_{i, k}\right]$. If detections are not independent, then detectors are competing and this can be described by assuming $\left[\boldsymbol{\omega}_{i,, k} \mid \boldsymbol{x}_{i, k}, s_{i, k}\right]$ has a multinomial distribution. This can occur, for example, when physical trapping of the individual in one detector removes the possibility of being captured in another detector for that occasion.

In surveys that employ the robust design approach (Pollock, 1982), the occasion, as discussed here, corresponds to the primary occasion. These primary occasions may include multiple secondary occasions. In that case, the above describes the likelihood contribution for each secondary occasion and, assuming secondary occasions to be independent, $\left[\boldsymbol{\omega}_{i, \cdot k} \mid \boldsymbol{x}_{i, k}, s_{i, k}\right]$ is a product of these terms.

\subsection{Open population model}

Two popular models in open population capture-recapture are the Cormack-Jolly-Seber (CJS) and the Jolly-Seber (JS) model (Schwarz and Arnason, 1996). For CJS, individuals are known to be alive from the first occasion they are studied: individuals can only This article is protected by copyright. All rights reserved. 
have life states that are 'alive' or 'dead'. Individuals may be first captured on different occasions and so tracked over different periods of time. For simplicity, it is assumed here that all individuals are tracked from occasion 1 onwards; however, the method is easily adapted to the case where individuals are tracked from different starting points. The probability that an individual alive in the study region in occasion $k$ survives until occasion $k+1$ is called the survival probability on occasion $k$ and is denoted $\phi_{k}$. In particular, only the capture histories of those individuals that are marked, captured at least once, are used and so the population studied, the population of marked individuals, has known size.

For JS, individuals can be born, as well as survive and die, during a survey. Furthermore, JS models assume unmarked individuals are exchangeable with marked individuals, that is, their capture histories arise from the same model. This assumption allows JS models to estimate the number of individuals ever to have lived during the survey, $N$. In SCR, individuals with activity center $\boldsymbol{x}$ arise from a Poisson process with rate $D(\boldsymbol{x})$. This rate is interpreted as the density at location $\boldsymbol{x}$. When incorporated with the JS model, this interpretation changes. Each individual is assigned an initial activity center, just as before, at location $\boldsymbol{x}$ with rate $D(\boldsymbol{x})$, but that individual is, possibly, only alive for some number of occasions.

Conceptually, all individuals are placed in a queue: some wait to enter the queue ('unborn'), some are in the queue ('alive'), and some have left the queue ('dead'). The parameter $N$ is the number who were in the queue at some point. The $N$ individuals are divided among the $k$ occasions: some are in the queue from occasion 1 , some join in occasion 2, and so on. The probability that an individual is selected to join the queue in occasion $k$ is $\alpha_{k}$. For example, $\alpha_{1}$ is the probability that an individual is in the queue at the beginning of the survey, that is, is alive from the beginning; $\alpha_{2}$ is the probability that an individual is born in occasion 2. Equivalently, the probability that an individual enters the queue on occasion $k$ given it has not entered up to that time is denoted $\beta_{k}$ where $\beta_{1}=\alpha_{1}$ and $\beta_{k}=\alpha_{k} / \prod_{l=1}^{k-1}\left(1-\beta_{l}\right)$ for $k>1$. Similarly, individuals leave the queue after occasion $k$ with probability $1-\phi_{k}$.

This queue can be described by a Markov chain. The life state of an individual in an occasion depends only on its state in the previous occasion, satisfying the Markov property. A Markov chain is fully specified by its initial distribution and transition probability matrix.

For JS, the initial distribution depends on an estimable parameter: $\boldsymbol{\delta}=\left(1-\alpha_{1}, \alpha_{1}, 0\right)$.

This article is protected by copyright. All rights reserved. 
The transition probability matrix for JS is

$$
\boldsymbol{\Gamma}_{k}=\left(\begin{array}{ccc}
1-\beta_{k} & \beta_{k} & 0 \\
0 & \phi_{k} & 1-\phi_{k} \\
0 & 0 & 1
\end{array}\right) \text { unborn }
$$

Note that, $\beta_{k}$, not $\alpha_{k}$, is used because it is the conditional probability of being born, given the individual hasn't been born up to that time, that is needed. This implicitly uses the knowledge that an individual in the 'unborn' state must have been in this state for all past occasions.

For CJS, the initial distribution $\boldsymbol{\delta}$ is known, all individuals begin in the alive state: $\boldsymbol{\delta}=$ $(1,0)$. The transition probability matrix for CJS is the $2 \times 2$ sub-matrix corresponding to the alive and dead states.

\subsection{Hidden Markov Model}

Hidden Markov models are applied to time series data where observed records at each time point depend on an unobserved Markov chain. Here, the capture history of an individual is recorded over time, and each capture record depends on the unobserved life state of that individual, where life states follow a Markov chain (Figure 1). Therefore, CJS and JS are examples of hidden Markov models.

[Figure 1 about here.]

Incorporating SCR with this formulation is simple. Conceptually, for each occasion, individuals in the queue (those that are alive) are either recorded or not depending on their activity center. Following hidden Markov model methodology, this is formulated by a matrix, termed here the detection matrix. The detection matrix for CJS and JS, $\boldsymbol{P}_{k}$, is a $2 \times 2$ and $3 \times 3$ diagonal matrix respectively where the $s^{\text {th }}$ diagonal is $\left[\boldsymbol{\omega}_{i, \cdot, k} \mid \boldsymbol{x}_{i, k}, s_{i, k}=s\right]$ for $s=$ unborn, alive, dead.

The power of recognising this to be a hidden Markov model is clear when it comes to averaging over all possible life histories, weighting each by their probability. It is simply a matrix product: $\left[\boldsymbol{\Omega}_{i} \mid \boldsymbol{x}_{i, 1}, \ldots, \boldsymbol{x}_{i, K}\right]=\boldsymbol{\delta} \boldsymbol{P}_{i, 1} \boldsymbol{\Gamma}_{1} \boldsymbol{P}_{i, 2} \boldsymbol{\Gamma}_{2} \ldots \boldsymbol{\Gamma}_{K-1} \boldsymbol{P}_{i, K} \boldsymbol{\epsilon}$ where $\boldsymbol{P}_{i, k}=\boldsymbol{P}_{k}\left(\boldsymbol{x}_{i, k}\right)$ and $\boldsymbol{\epsilon}$ is a column vector of ones with the appropriate size.

\subsection{Spatial process}

Until now, the activity center of each individual has been treated as known. Each individual is associated with a latent movement path: its activity centers over all occasions. This article is protected by copyright. All rights reserved. 
In the standard implementation of maximum likelihood spatial capture-recapture, each individual's activity center is assumed to be stationary, that is, $\boldsymbol{x}_{i, k}=\boldsymbol{x}_{i, 1}$ for all $k$; a spatial point process model is specified for the initial activity center. The latent activity centers are then marginalised by a two-dimensional numerical integration for each individual. In the Bayesian framework, correlated movement of activity centers over occasions has been implemented; the corresponding maximum likelihood method to do so is presented here.

Activity center movements are assumed to be independent random deviates from a two-dimensional Gaussian distribution: $\boldsymbol{x}_{i, k+1}$ conditional on $\boldsymbol{x}_{i, k}$ is a two-dimensional Gaussian random variable with mean $\boldsymbol{x}_{i, k}$ and variance $\nu^{2} \Delta t_{k} \boldsymbol{I}$ where $\Delta t_{k}$ is the number of time units separating occasions $k$ and $k+1$, and $\boldsymbol{I}$ is an identity matrix. The variable $\nu$ is termed the movement range. This is a Markov process. It follows that the probability density function (PDF) of the movements for a single individual $i$ given their initial activity center is a product of bivariate Gaussian PDFs, $\left[\boldsymbol{x}_{i, 2}, \ldots, \boldsymbol{x}_{i, K} \mid \boldsymbol{x}_{i, 1}\right]$. The full PDF for the movement of activity centers is thus specified once a spatial model is determined for the initial activity center. The appropriate spatial point process model depends on whether a CJS or JS model is used.

\subsection{Likelihood}

The point process from which the initial location of each individual arises is assumed to be an inhomogeneous Poisson process with rate $D(\boldsymbol{x})$ at location $\boldsymbol{x}$. The distribution of initial locations of individuals detected at least once during the survey is thus a thinned point process with thinning probability $p\left(\boldsymbol{x}_{1}\right)=1-\left[\boldsymbol{\Omega}_{0} \mid \boldsymbol{x}_{1}\right]$ where $\boldsymbol{x}_{1}$ is the initial activity center of the individual and $\Omega_{0}$ is the capture history of an individual that is never seen by any detector on any occasion with $\left[\boldsymbol{\Omega}_{0} \mid \boldsymbol{x}_{1}\right]=\int\left[\boldsymbol{\Omega}_{0} \mid \boldsymbol{x}_{1}, \ldots, \boldsymbol{x}_{K}\right]\left[\boldsymbol{x}_{2}, \ldots, \boldsymbol{x}_{K} \mid \boldsymbol{x}_{1}\right] \mathrm{d} \boldsymbol{x}_{2} \ldots \mathrm{d} \boldsymbol{x}_{K}$. Thus, the overall intensity function of the thinned point process is $\mu(\boldsymbol{x})=D(\boldsymbol{x}) p(\boldsymbol{x})$ for initial activity center $\boldsymbol{x}$. The likelihood is built from two components: the probability of detecting $n$ individuals and the probability of observing the capture histories given detection at least once, averaged over activity centers.

The likelihood, $\mathcal{L}$, assuming individuals to be independent, can now be stated. For JS, the likelihood is

$$
\begin{aligned}
\mathcal{L}=\frac{\exp (-\bar{\mu})}{n !} \prod_{i=1}^{n} \int & {\left[\boldsymbol{\Omega}_{i} \mid \boldsymbol{x}_{i, 1}, \ldots, \boldsymbol{x}_{i, K}\right]\left[\boldsymbol{x}_{i, 2}, \ldots, \boldsymbol{x}_{i, K} \mid \boldsymbol{x}_{i, 1}\right] } \\
& \times D\left(\boldsymbol{x}_{i, 1}\right) \mathrm{d} \boldsymbol{x}_{i, 1} \ldots \boldsymbol{x}_{i, K}
\end{aligned}
$$

where $\bar{\mu}=\int \mu(\boldsymbol{x}) \mathrm{d} \boldsymbol{x}$. A full derivation is given in the Web Appendix A.

This article is protected by copyright. All rights reserved. 
For CJS, data are collected conditional on $n$ individuals, thus the thinned Poisson process is an inhomogeneous Binomial point process. The likelihood is thus

$$
\begin{aligned}
\mathcal{L}=\frac{1}{\bar{D}^{n}} \prod_{i=1}^{n} \int & {\left[\boldsymbol{\Omega}_{i} \mid \boldsymbol{x}_{i, 1}, \ldots, \boldsymbol{x}_{i, K}\right]\left[\boldsymbol{x}_{i, 2}, \ldots, \boldsymbol{x}_{i, K} \mid \boldsymbol{x}_{i, 1}\right] } \\
& \times D\left(\boldsymbol{x}_{i, 1}\right) \mathrm{d} \boldsymbol{x}_{i, 1} \ldots \boldsymbol{x}_{i, K}
\end{aligned}
$$

where $\bar{D}=\int D(\boldsymbol{x}) \mathrm{d} \boldsymbol{x}$.

In both cases, the likelihood can be decomposed into a product of $2 K$-dimensional integrals, one for each individual. When the activity center of each individual is assumed to be the same over all occasions, stationary, then this reduces to a product of twodimensional integrals, one for each individual, and can be computed using standard, numerical integration techniques. When the activity center of an individual moves, as described above, according to a Gaussian process, the integral can be computed efficiently by incorporating the numerical integration with the HMM formulation. As with standard numerical quadrature, the survey area is divided into $M$ grid points, termed the mask or mesh in the SCR literature. Each individual is assumed to have an activity center that resides at one of these grid points. In other words, the grid point representing the activity center of individual $i$ on occasion $k, g_{i, k}$, is a latent variable. The movement of the individual across the grid is a hidden process. Assuming movement is a Markov process implies that the combined hidden state $\left(g_{i, k}, s_{i, k}\right)$ is a Markov process. Transition between life states is described in Section 2.2. Transitions between grid points can similarly be formulated as a transition matrix. The probability of moving from grid point $a$ to grid point $b$ is denoted $\gamma_{a, b}$. Given a Gaussian movement process, the transition probability density of movement to a point $\boldsymbol{y}$ conditional on being located at a point $\boldsymbol{x}$ is a bivariate Gaussian PDF with mean $\boldsymbol{x}$ and variance $\nu^{2} \boldsymbol{I}$, for identity matrix $\boldsymbol{I}$. The probability of transition from location $\boldsymbol{x}$ to the grid cell $B$, represented by grid point $b$, is the integral of this transition density over $B$. The entry $\gamma_{a, b}$ is the transition probability from point $\boldsymbol{x}$ to grid cell $B$ averaged over all $\boldsymbol{x}$ in the grid cell surrounding grid point $a$. For each grid point $g_{i, k}$ corresponding to spatial location $\boldsymbol{x}_{i, k}$, Section 2 gives the initial life state distribution $\boldsymbol{\delta}$, transition probability matrix $\boldsymbol{\Gamma}_{k}$ and the detection matrix $\boldsymbol{P}_{i, k}$. The corresponding entries for the augmented hidden process $\left(g_{i, k}, s_{i, k}\right)$, the initial state vector $\boldsymbol{\Delta}$, the transition probability matrix $\widetilde{\boldsymbol{\Gamma}}_{k}$, and the detection matrix $\widetilde{\boldsymbol{P}}_{i, k}$ can be computed from these (see Web Appendix B). Given this, the likelihood approximated by numerical integration over all activity center movements can be computed by a HMM likelihood: $\mathcal{L}=\prod_{i=1}^{n}\left\{\boldsymbol{\Delta}\left(\prod_{k=1}^{K-1} \widetilde{\boldsymbol{P}}_{i, k} \widetilde{\boldsymbol{\Gamma}}_{k}\right) \widetilde{\boldsymbol{P}}_{i, K} \boldsymbol{\epsilon}\right\}$ where $\boldsymbol{\epsilon}$ is a column vector of ones of

This article is protected by copyright. All rights reserved. 
appropriate size. In practice, the matrix $\widetilde{\boldsymbol{\Gamma}}_{k}$ is a sparse matrix when $\nu$ is small compared to the size of the grid: individuals move small distances compared to the size of the survey region. Thus, efficient sparse matrix computations can be used to perform the matrix multiplication.

Maximum likelihood estimation can be used to obtain point, variance and interval estimates of all parameters in the standard way with Wald or profile likelihood intervals; similarly, priors can be specified for parameters and MCMC used with this semi-complete data likelihood.

\subsection{Abundance}

For JS models, the total number of individuals to have lived at some time during the survey is estimated: $\widehat{N}=\int \widehat{D}(\boldsymbol{x}) \mathrm{d} \boldsymbol{x}$. This quantity, however, is rarely of interest. The true interest lies in estimating abundance over time. The mean estimated density in occasion $k$ can be computed: $\widehat{D}_{k}(\boldsymbol{x})=\widehat{D}(\boldsymbol{x}) \widehat{\boldsymbol{\delta}}_{1} \ldots \widehat{\Gamma}_{k-1} \boldsymbol{\epsilon}_{1}$, where $\boldsymbol{\epsilon}_{1}$ is the $3 \times 1$ vector $(0,1,0)$. The estimated abundance on occasion $k$ is then derived in the usual way: $\widehat{N}_{k}=\int \widehat{D}_{k}(\boldsymbol{x}) \mathrm{d} \boldsymbol{x}$.

When parameters are estimated by maximum likelihood, the variance and confidence intervals for $\widehat{N}_{k}$ can be obtained assuming a log-normal asymptotic distribution (Fewster and Jupp, 2009) or by bootstrap. If Bayesian estimation were used, posterior inference for $N_{k}$ is obtained trivially from the above formula and the posteriors of the model parameters.

\section{Application: Jaguars}

The aim is to infer the population size and demographics over time of male jaguars in the east of the Cockscomb Basin Wildlife Sanctuary, Belize (Harmsen et al., 2010) in a survey area of 1598 square kilometers. Capture-recapture camera trap surveys were conducted for on average 91 days each year between 2002 - 2008 and 2011 - 2015. Full details of the survey are given by Harmsen et al. (2017).

Each survey year was considered an occasion and capture histories comprised counts of the number of times each jaguar was seen on each camera trap. A total of 21 pairs of cameras were used at some time during the survey, each pair being treated as a single detector. Nineteen of the detectors remained in the same position over the entire survey (Figure 2). Detectors were placed at approximately two kilometer intervals along trails preferentially used by male jaguars. In 2011, photographic cameras were replaced with digital technology, which may affect detectability.

This article is protected by copyright. All rights reserved. 
[Figure 2 about here.]

\subsection{Data summary}

Over 12 occasions, 53 unique male jaguars were detected with an average of 23.4 detections per individual. The biological research focusses on three processes: detection, recruitment, and survival. Before considering a statistical model, summary statistics can be computed. These summary statistics are not rigorous parameter estimates. Nevertheless, they are useful because they provide independent pictures of the data. Open population models and spatial capture-recapture models induce non-linear relationships between parameters: a larger estimated detection range may force a reduced estimated encounter rate, for example. The resultant estimates from a model are a compromise between the accurate depiction of the detection, demographic, and movement processes in light of the data collected. Summary statistics allow the analyst to determine before modeling what an appropriate model is and to identify if the constraints of the model have distorted the resulting inference, leading to a poor goodness-of-fit.

- Detection range: The detection range, the range covered within each occasion by an individual, can be summarised by the statistics proposed by Calhoun and Casby (1958) (CC statistic): $C_{\text {within }, k}=\sqrt{\sum_{r=1}^{R}\left\|\boldsymbol{y}_{r, k}-\overline{\boldsymbol{y}}_{k}\right\|^{2} /(2 R-2)}$ where $\|\cdot\|$ denotes the Euclidean distance metric, $R$ is the total number of sightings of the individual during occasion $k, \boldsymbol{y}_{r, k}$ is the location of the detector the individual was seen at during occasion $k$ for sighting $r$, and $\overline{\boldsymbol{y}}_{k}$ is the mean of these locations over occasion $k$. This statistic provides an initial estimate for the detection range. In this survey, the $C_{\text {within }}$ statistic indicates a mean individual range of 3120 meters with a standard deviation of 408 meters. Individuals captured in 2005 had the smallest mean range (2459 meters) and those in 2013 the largest range (4069 meters). It should be noted that these are the detected ranges; movements beyond the scope of the detectors is unobserved.

- Encounter rate: The encounter rate, $\lambda_{0}$, is the number of encounters an individual has with a detector when its activity center coincides with the location of that detector. The encounter rate (ER) summary statistic is chosen to reflect this quantity. For occasion $k, E R_{k}=\frac{1}{n} \sum_{i=1}^{n} \max _{j} \omega_{i, j, k} / T_{k}$ where $T_{k}$ is the number of days occasion $k$ lasted. This statistic gives a negatively-biased estimator of encounter rate, but provides a useful indicator of change in encounter rate over time. Here, the mean ER across occasions was 0.046 individuals per day with a standard deviation of 0.012 . The ER statistic has a mean of 0.040 before 2011, when the digital cameras were first used, This article is protected by copyright. All rights reserved. 
and this increases to 0.055 from 2011. This indicates that the change in technology may have affected the detection process.

- Survival: The survival process is a geometric process in which individuals fail to survive with probability $1-\phi$ on each occasion. A useful summary statistic is the mean number of occasions between each individual's first and last sighting for those individuals seen on at least two occasions. Here, on average, the duration between the first and last sighting of an individual is 3.49 years. For a geometric distribution, this corresponds to an annual survival probability of 0.71 .

- Recruitment: Individuals are recruited by occasion. We use the proportion of detected individuals that were first seen on each occasion as a recruitment-related summary statistic. In this survey, $19 \%$ of individuals were sighted in the first occasion and there was no apparent trend in number after that, with on average $7.4 \%$ newly sighted individuals per occasion.

- Movement: Activity centers may move during the survey. It is important to investigate whether this occurs. One method is to compute the $\mathrm{CC}$ statistic over the entire survey, $C_{\text {all }}$, where the occasion-specific mean $\overline{\boldsymbol{y}}_{k}$ is replaced by the mean detection location over the entire survey $\overline{\boldsymbol{y}}$. This can be compared to the mean withinoccasion CC statistic computed above, $C_{\text {within }}$. For stationary activity ranges, the two will be similar; for moving activity centers, the range covered by an individual over the survey will be larger than that covered in any single occasion. Here, $C_{\text {all }}$ across the entire twelve survey years is 4512 kilometers; this is on average 1500 meters larger than $C_{\text {within, }}$, suggesting that movement has occurred. We can define $C_{\text {between }}$ to be the CC statistic for movement of activity centers between occasions, such that $C_{\text {all }}^{2}=C_{\text {between }}^{2}+C_{\text {within }}^{2}$. Here, the mean between-occasion range is 3204 meters with a standard deviation of 460 meters. This estimate is biased by the detection process and population dynamics, but can be used as an exploratory diagnostic. Here, there is indication that a model with movement is likely to be required as the between-occasion range is similar to the within-occasion range.

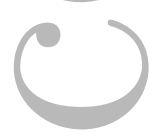

3.2 Model

SCR Jolly-Seber models with and without moving activity centers were fit to the data by maximum likelihood where variance was estimated using the inverse Hessian approach. The integration was performed over a grid, often called a mesh, with a mean buffer distance around the trapping area of 17 kilometers; stability of the integration on the This article is protected by copyright. All rights reserved. 
mesh was investigated as in the mask.check function of the secr software package (Efford, 2012), see the Web Appendix C for details.

Models had three components:

- Detection model: It is assumed that the number of encounters of individual $i$ with trap $j$ was Poisson distributed with a mean given by $\lambda_{j, k}\left(\boldsymbol{x}_{i, k}\right.$, alive $)=\lambda_{0, k} \exp \left\{-d_{j}\left(\boldsymbol{x}_{i, k}\right)^{2} /\left(2 \sigma_{k}^{2}\right)\right.$ where $\boldsymbol{x}_{i, k}$ is the activity center of individual $i$ on occasion $k$ and $d_{j}(\boldsymbol{x})$ is the distance between detector $j$ and location $\boldsymbol{x}$. The parameter $\lambda_{0, k}$ is termed the base encounter rate and $\sigma_{k}$ the detection range on occasion $k$.

- Open population model: The Schwarz-Arnason Jolly-Seber model (Schwarz and Arnason, 1996) is assumed with recruitment probabilities $\beta_{1}, \ldots, \beta_{K}$ for $K$ occasions and survival probability $\phi_{k}$ between occasions $k$ and $k+1$. When survival probability is constant, $\phi$, the survival probability between occasions $k$ and $k+1$ was formulated as $\phi_{k}=\phi^{\delta_{k}}$ where $\delta_{k}$ is the duration between the end of occasion $k$ and the beginning of occasion $k+1$ : longer durations between occasions lead to lower survival probabilities between occasions. Here, time was defined in years and $\phi$ is estimated annual survival.

- Spatial process: Initial activity centers are assumed to be distributed according to a homogeneous Poisson process with constant rate $D$. For models with moving activity centers, activity centers move according to a Gaussian process with standard deviation $\nu_{k}$ between occasions $k$ and $k+1$. Movement is approximated by movement on the mesh, that is, it is assumed each individual's activity centers during the survey are contained within the mesh.

Models were considered where parameters may change between occasions or be constant, except $D$ which was a constant. For the detection model, a covariate specifying whether a digital or photographic camera was used is also considered. Models were compared by AIC.

[Table 1 about here.]

The best fitting model selected by AIC had activity centers, encounter rate, and detection range change by occasion while survival probability, recruitment rate, and movement range were constant (Table 1). The covariate describing the technology used for each camera (digital or not) did not provide a better or comparable fit (assessed by AIC) compared to allowing detection probability to change for every occasion.

The best fitting model selected by AIC among models where activity centers were stationary over time also had encounter rate and detection range vary by occasion while all other parameters were constant. The estimated trend in density for the stationary This article is protected by copyright. All rights reserved. 
model and model with moving activity centers is shown in Figure 3. Table 1 shows the maximum likelihood estimates from both models. There is a remarkable difference in the estimated mean abundance: a 40\% reduction in the estimated abundance when activity centers are assumed to be stationary. Furthermore, the stationary model estimates survival to be $10 \%$ lower and activity range to be $60 \%$ larger. The substantial effect the assumption of stationary activity centers has on the resultant inference makes clear that this assumption must be validated for any application of open population models. Moving activity centers, when present in the survey but unaccounted for in the model, leads to two problems. First, as individuals are seen over a wider range over the entire survey compared to within each occasion, the stationary model compensates for this by overestimating activity range and underestimating encounter rate; this ultimately leads to an overestimation of detection probability and, in this case, a substantially underestimated density. The second problem is while the model with movement is able to account for reduced detectability due to movement, the stationary model cannot and tends to explain this reduced detectability by lowering survivial probability: individuals that move out of detectable range are best explained by having failed to survive.

One question is whether there is evidence that population density has changed over time. To investigate this, one can consider the estimated rate of change in density between occasions: $\Delta_{k}=\left(D_{k+1}-D_{k}\right) / \Delta t_{k}$ where $\Delta t_{k}$ is the time between occasions $k$ and $k+1$. Using the best-fitting model with moving activity centers, a parametric bootstrap, simulating from the estimated asymptotic distributions of the parameters, is used to estimate the variance of these differences. The mean change in density between occasions was 0.06 individuals per 100 square kilometers with an estimated $95 \%$ confidence interval of $(-0.02,0.21)$ using 1000 bootstrap re-samples. As the estimated confidence interval includes zero, this indicates that the evidence for population density changing over time is not statistically significant.

[Figure 3 about here.]

\subsection{Comparison with other methods}

Two popular SCR alternatives to the HMM method presented here are closed-population SCR and the Bayesian implementation of the open population models using data augmentation. These methods were applied to the jaguar case study to compare resultant inference and efficiency. For both methods, the same formulations for detection and, for the Bayesian model, population dynamics were used as in the models above.

For the year-specific closed-population SCR approach, the population was assumed to This article is protected by copyright. All rights reserved. 
be closed within the survey conducted each year and surveys across years assumed to be independent. This means that if an individual was captured in separate years, this information is ignored and the detections treated as if they are from distinct individuals. Hence, one cannot explicitly estimate recruitment or survival probability. On the other hand, as activity centers between years are unrelated, this model is robust to movement between occasions. Separate detection and density parameters are estimated for each year using standard SCR methods. Figure 3 shows that the open population model with moving activity centers and the closed population approach provide similar inference on trend over time; however, the trend in density is more noisy when estimated by closed population SCR due to treating years separately. To mitigate this, density can be described by a smooth, such as a cubic spline, over time. Closed-population models were fitted with density constant or changing smoothly according to a regression spline with increasing dimension and then compared by AIC. The best model describes density as a constant over time with mean 2.33 individuals per 100 square kilometers and $95 \%$ confidence interval $(1.94,2.80)$. This is consistent with the open population model where no statistically significant evidence is found for a change in density over time.

The closed population approach led to less precise estimates of density. The open population model provides more precise estimates by using the additional information that the closed population approach ignores. Applying a smooth to the closed population estimates leads to similar precision to that from the open population model. The primary advantage of the open population model over the closed population model is the ability to make explicit inference about survival and recruitment probabilities from the observations made on individuals during the survey.

The HMM approach was also compared to the Bayesian data augmentation approach proposed in Gardner et al. (2010). The Bayesian method was implemented in rjags 4.6 (Plummer, 2013) with weakly informative priors. The posterior was approximated by 100, 000 iterations with a burn-in of 5000 iterations for the stationary model and 20,000 for the model with moving activity centers. Inference obtained from the Bayesian method was similar to that from the maximum likelihood HMM approach for both stationary and moving activity center models (see Web Appendix D for the Bayesian results).

For the simplest model, all parameters being constant over time, maximum likelihood inference was obtained in 5.5 minutes while the data augmentation took 23 hours. For the best fitting models identified above, maximum likelihood inference took 4 hours to fit compared to 1.5 days. Both methods were implemented on a desktop Intel(R) Core(TM) i7-4790 CPU (@3.6 GHz) with a cache size of 8 Gb and 16 Gb of RAM. Overall, compared

This article is protected by copyright. All rights reserved. 
with data augmentation in JAGS, averaging over life history with $\mathrm{R}$ and Rcpp using a HMM was more computationally efficient, provided similar inference, and avoided auto-correlation issues common when MCMC is applied to models with latent temporal processes. Some of this computational efficiency is gained from switching from using JAGS to using Rcpp and some from switching from data augmentation to marginalization. The comparison, however, does show the relative efficiency of the maximum likelihood analysis compared to the common, at present, JAGS analysis conducted by practitioners.

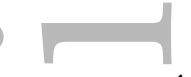

\section{Simulation study}

The performance of the maximum likelihood estimators was investigated by simulation. Surveys identical to the jaguar survey, with the same camera locations and number of occasions, were simulated. All parameters were set to be constant over the survey with $\lambda_{0}=0.05, \sigma=3000, \phi=0.8, \beta_{1}=0.2$, and $D=0.06$. Two scenarios were considered, one where activity centers were stationary and one where they moved by Brownian motion with standard deviation $\nu=3000$. For the stationary simulations, an open population model with stationary activity centers and a closed population spatial capture-recapture model were fit to each data set by maximum likelihood. For the simulation with moving activity centers, two open population models were considered, one with a movement model and one without, along with a closed population model. Percentage relative

bias and $95 \%$ confidence interval coverage was estimated for each parameter from one thousand simulations.

When activity centers are stationary, the model assuming stationary activity centers is negligibly biased for all parameters (Figure 4) and $95 \%$ confidence interval coverage is $94.6 \%$ on average for all parameters. When simulated activity centers move between occasions, detection, survival, and recruitment parameters become notably biased (Figure 4). Despite this, the estimated bias for the super-population density remains small. This is similar to the findings of Royle et al. (2016): the stationary model provides robust estimates of density by using the detection model to account for the unobserved, unmodeled movement process: detection range is overestimated and encounter rate underestimated. In the open population model, both the detection and population dynamics models are distorted to account for the movement of individuals in to, within, and out of the study area: initial recruitment probability, $\beta_{1}$, and survival probability are underestimated: individuals not present initially immigrate and some present, emigrate.

In this case, however, interest is not in the super-population density estimate, but in the This article is protected by copyright. All rights reserved. 
estimated abundance (or density) within each occasion. As in the jaguar application, the estimated density for each occasion is negatively biased when movement is not accounted for. The within-occasion density estimator is biased because the population dynamics (survival and recruitment) are confounded with the unmodeled movement process.

The open population model where movement is explicitly modeled provides estimates that are negligibly biased $(<3 \%$ in all cases) and confidence interval coverage was less than one percent from nominal. For this scenario, $\beta_{1}$ was marginally underestimated and $\bar{N}$ marginally overestimated. This may indicate that at low sample sizes, moving activity centers can still cause bias in the detection or population dynamics models due to the subtle confounding between these processes.

When compared to the closed-population model fit to the same simulated data sets, the mean percentage relative root-mean-square error in the estimate of density for each occasion was on average $30 \%$ smaller for the open population model when compared to the closed population model. Confidence intervals for the density on each occasion were on average 2.5 times wider using the closed population model compared to the open population model. The root-mean-square error and uncertainty in density estimators from the closed population model could be reduced using a smoothing approach. When smoothed, closed population models can provide similar inference on the trend in population density, but not the mechanisms that drive these trends.

[Figure 4 about here.]

\section{Conclusion}

Formulating open population spatial capture-recapture (SCR) as a hidden Markov model (HMM) incorporates CJS and JS models into a general framework and allows marginalization over life histories and movement to be achieved with computational efficiency, making more complex modeling and model selection practically feasible. Maximum likelihood estimation leads to similar inference compared with a data augmentation, Bayesian approach; furthermore, marginalization could be used in the Bayesian context with a semi-complete data likelihood (King et al., 2016). Overall, the presented framework is flexible and open to extension: alternative movement models, life histories with more states (e.g. temporary emigration), and incorporation of observed states (i.e. dead recoveries or recorded births).

This article is protected by copyright. All rights reserved. 


\section{Acknowledgements}

The authors thank the associate editor and two anonymous reviewers for their insightful

comments that led to an improved paper. David Borchers was part-funded by EPSRC grant EP/K041061/1 and Richard Glennie was funded by the Carnegie Trust.

\section{References}

Borchers, D. L. and Efford, M. (2008). Spatially explicit maximum likelihood methods for capture-recapture studies. Biometrics 64, 377-385.

Calhoun, J. B. and Casby, J. U. (1958). Calculation of home range and density of small mammals. Number 55. US Department of Health, Education, and Welfare, Public Health Service.

Chandler, R. B. and Clark, J. D. (2014). Spatially explicit integrated population models. Methods in Ecology and Evolution 5, 1351-1360.

Cormack, R. (1964). Estimates of survival from the sighting of marked animals. Biometrika 51, 429-438.

Efford, M. (2004). Density estimation in live-trapping studies. Oikos 106, 598-610.

Efford, M. (2012). secr: Spatially explicit capture-recapture models. R package version 3.2.0.

Fewster, R. and Jupp, P. (2009). Inference on population size in binomial detectability models. Biometrika 96, 805-820.

Gardner, B., Reppucci, J., Lucherini, M., and Royle, J. A. (2010). Spatially explicit inference for open populations: estimating demographic parameters from cameratrap studies. Ecology 91, 3376-3383.

Harmsen, B. J., Foster, R. J., Sanchez, E., Gutierrez-González, C. E., Silver, S. C., Ostro, L. E., et al. (2017). Long term monitoring of jaguars in the Cockscomb Basin wildlife sanctuary, Belize; implications for camera trap studies of carnivores. PloS one 12, e0179505.

Harmsen, B. J., Foster, R. J., Silver, S. C., Ostro, L. E., and Doncaster, C. P. (2010). The ecology of jaguars in the Cockscomb Basin wildlife sanctuary, Belize. The biology and conservation of wild felids pages 403-416.

Jolly, G. M. (1965). Explicit estimates from capture-recapture data with both death and immigration-stochastic model. Biometrika 52, 225-247.

King, R., McClintock, B. T., Kidney, D., and Borchers, D. L. (2016). Capture-recapture abundance estimation using a semi-complete data likelihood approach. Annals of Applied Statistics 10, 264-285.

This article is protected by copyright. All rights reserved. 
Otis, D. L., Burnham, K. P., White, G. C., and Anderson, D. R. (1978). Statistical inference from capture data on closed animal populations. Wildlife monographs pages $3-135$.

Plummer, M. (2013). rjags: Bayesian graphical models using MCMC. R package version 3,

Pollock, K. H. (1982). A capture-recapture design robust to unequal probability of capture. The Journal of Wildlife Management 46, 752-757.

R Core Team (2017). R: A Language and Environment for Statistical Computing. R Foundation for Statistical Computing, Vienna, Austria.

Royle, J. A., Chandler, R. B., Sollmann, R., and Gardner, B. (2013). Spatial capturerecapture. Academic Press.

Royle, J. A., Fuller, A. K., and Sutherland, C. (2016). Spatial capture-recapture models allowing markovian transience or dispersal. Population ecology 58, 53-62.

Schwarz, C. and Arnason, A. (1996). A general methodology for the analysis of capturerecapture experiments in open populations. Biometrics 52, 860-873.

Seber, G. A. (1965). A note on the multiple-recapture census. Biometrika 52, 249-259.

Zucchini, W., MacDonald, I. L., and Langrock, R. (2016). Hidden Markov models for time series: an introduction using $R$, volume 150 . CRC press.

\section{Supporting Information}

Web Appendices referenced in Sections 2.5, 3.2, and 3.3 are available with this paper at the Biometrics website on Wiley Online Library. Also, the R (R Core Team, 2017) package, openpopscr, is available on GitHub: http://github.com/r-glennie/openpopscr.

This article is protected by copyright. All rights reserved. 


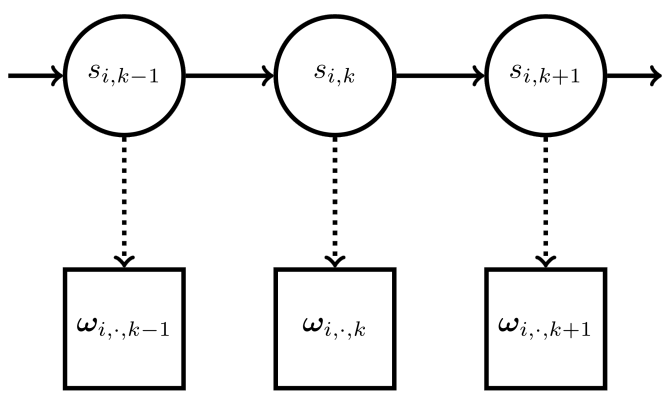

Figure 1. HMM formulation of open population spatial capture-recapture model: individual $i$ has life state $s_{i, k}$ and capture record (over all detectors) $\boldsymbol{\omega}_{i, \cdot, k}$ in occasion $k$. Life history is a hidden Markov process and capture records are independent conditional on life history. Nodes represent random variables and arrows terminate on variables whose distribution is defined conditional on the variable from whence the arrow originated. Unconnected nodes are conditionally independent given their parent nodes.

This article is protected by copyright. All rights reserved. 


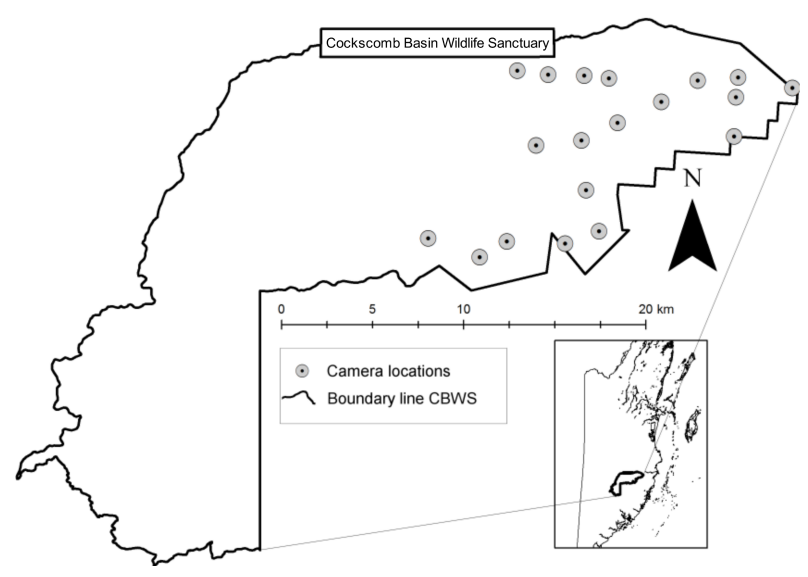

Figure 2. Cockscomb Basin Wildlife Sanctuary, Belize with approximate positions of camera traps during the survey.

This article is protected by copyright. All rights reserved. 


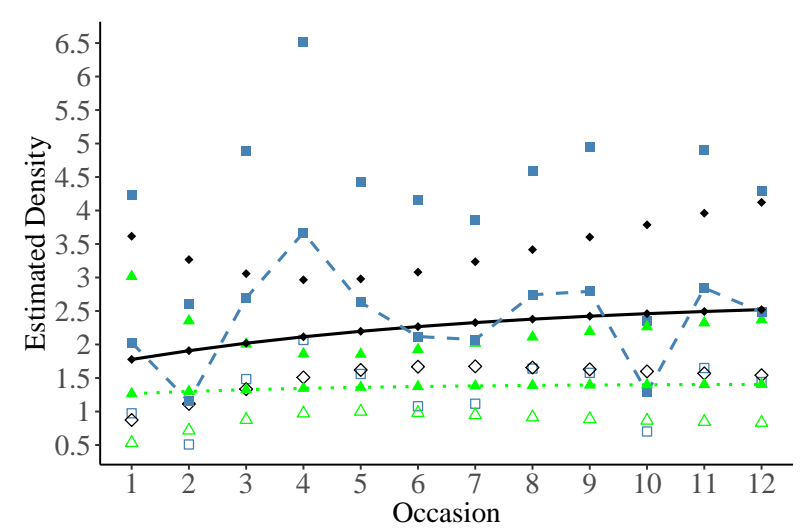

Figure 3. Estimated density per 100 square kilometers (line) of male jaguars in the Cockscomb Basin Wildlife Sanctuary, Belize for each occasion (points) with lower (unfilled) and upper (filled) 95\% confidence interval bounds for closed-population SCR model (dashed line, squares), open population maximum likelihood model with stationary activity centers (dotted line, triangles), and open population maximum likelihood with moving activity centers (solid line, diamonds). Models were selected as the best fit by AIC. All models have detection parameters vary by occasion and open population models have constant survival and recruitment probabilities. This figure appears in color in the electronic version of this article.

This article is protected by copyright. All rights reserved. 


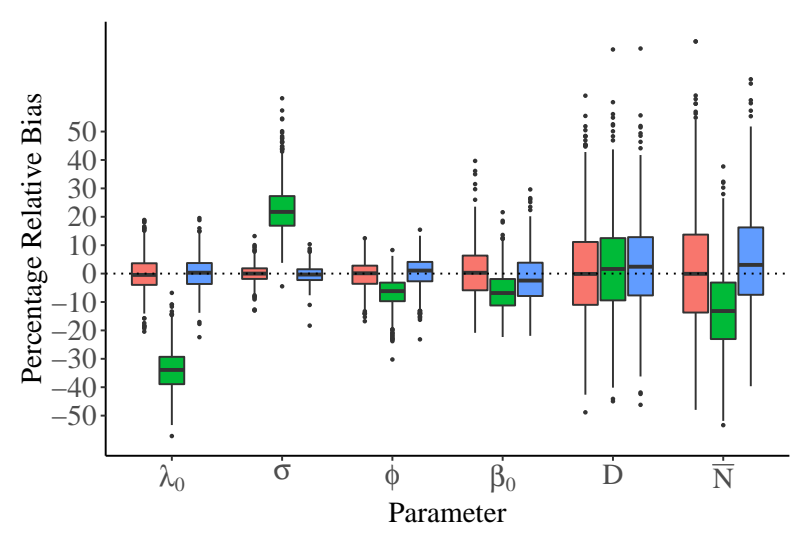

Figure 4. Boxplot of relative percentage bias from one thousand simulated open population spatial capture-recapture surveys for encounter rate $\lambda_{0}$, detection range $\sigma$, survival probability $\phi$, probability an individual is present at the start of the survey $\beta_{1}$, super population density $D$, and mean abundance on a single occasion $\bar{N}$ in the Cockscomb Wildlife Basin Sanctuary. For each parameter, bias is shown for the model assuming stationary activity centers fitted to data simulated with stationary activity centers (left) and moving activity centers (middle), and bias from a model allowing for activity center movement fit to data simulated with moving activity centers (right). This figure appears in color in the electronic version of this article.

This article is protected by copyright. All rights reserved. 
